\title{
Comparison of Body Mass Index and Cholelithiasis Diagnosed on Ultrasound in the Population of Pindi Bhattian
}

\author{
I. Mehmood, M. Uzair, A. Malik, Y. Gillani, D. E. Shahwar, Z. Ilyas and N. Fatima
}

\section{ABSTRACT}

Obesity is one of the emerging conditions in our population. Gallstones are composed of cholesterol. It is postulated that gallstones are associated with the deposition of cholesterol. Ultrasound is the first line imaging modality for the diagnosis of cholelithiasis. If gallstones are correlated with BMI, it will open up a new vista for further research.

In Yaqoob Ultrasound Clinic, PindiBhattian, Pakistan, a cross sectional analytical study was conducted. All included patients were inquired regarding variables like Age, Height, Weight, Presences of stones, Wall thickness of gall bladder, and location of gall stones on grey scale ultrasound findings. Patients were asked to lie down and expose their abdomen; grey scale abdominal ultrasound was performed of these patients. All variables mentioned above for each patient was recorded and maintain in their individual case record form (CFR). Data was collected during allocated period. Statically package SPSS version 2.4 was used to check the collected data and to organize and compile results. According to results out of total 160 patients, 105 were females and 55 were male. Out of 160 patients $150(93.8 \%)$ were present with gall stones and $10(6.3 \%)$ were having no gall stone. In total 61 patients $(38.1 \%)$ were having single gall stone whereas $89(55.6 \%)$ were diagnosed with multiple gall stones. According to the results, minimum age was 18 years and maximum was 80 years. Our study concluded that obesity is one of the major risk factors for gall stones. Obesity is usually more common in females, so they are more likely to have gall stones and overweight patients were more prone to GB stones.

Keywords: Body mass index, cholelithiasis, obesity, ultrasound.
Submitted : May 22, 2021

Published : October 20, 2021

ISSN: 2593-8339

DOI: $10.24018 /$ ejmed.2021.3.5.887

\section{Mehmood}

University of Lahore, Pakistan.

(e-mail: myfluffy123123@gmail.com)

M. Uzair

University of Lahore, Pakistan.

(e-mail: muhammaduzair@gmail.com)

A. Malik

University of Lahore, Pakistan.

(e-mail: adnanmalik@gmail.com)

Y. Gillani

University of Lahore, Pakistan.

(e-mail: yousafgillani@gmail.com)

D. E. Shahwar

University of Lahore, Pakistan.

(e-mail: dureshahwar@gmail.com)

Z. Illayas

University of Lahore, Pakistan.

(e-mail: zainabillayas@gmail.com)

N. Fatima

University of Lahore, Pakistan.

(e-mail: noorfatima@gmail.com)

\section{INTRODUCTION}

The human gallbladder is a pyriform sac that lies in the gallbladder fossa, a downturn situated inferiorly to the liver. It always lies in right upper quadrant, over the cross over colon, and is normally close to the duodenal cap overlying, however well anterior to, the right renal shadow. The human gallbladder is 7 to $10 \mathrm{~cm}$ long, 3 to 4 wides, in grown-ups. Roughly $50 \mathrm{ml}$ is the capacity of gall bladder. The wall thickness of GB is generally $2 \mathrm{~mm}$ and fluctuates with gallbladder distension. Free connecting tissues are connecting the gall bladder to the liver, which contains little veins and lymphatics associating the gallbladder to the liver. The rest of the gallbladder, which isn't in direct contact with the liver, is covered by peritoneum reflected from the liver. A cystic pipe is associated with the gall bladder that channels into the regular bile pipe (CBD), with no sphincter. The length of the cystic channel is 3-4 cm long and joins the common hepatic duct to form the CBD.
Symptomatology and presence of absence of complications helps us decide how to manage the cholelithiasis. In general, management is associated with multiple factors that includes healthy lifestyle and dietary modification and medication. Silent or incidental gallstones should be observed and managed expectantly. Pain control, antiemetics, and, if indicated, dissolution agents, all come under the pharmacological management [1], Oral dissolution therapy is a way in which we use bile acids to dissolve gallstones and it was successful in an extremely limited patient population. Gallstone reappearance is a hindrance of this treatment; roughly $25 \%$ of patients developed reappearing gallstones within five years. Currently, bile acid treatment is only opted for patients unsuitable or reluctant to go through a surgical procedure. Cholecystectomy stays the essential system for the administration of suggestive gallstone infection. It is protected, has the most minimal danger of repeat, and furnishes $92 \%$ of patients with complete alleviation of their biliary agony. Various benefits of 
Laparoscopic cholecystectomy compared with, and the open cholecystectomy includes better cosmesis, prior get back to work, lower costs, lower mortality, minimal postoperative pain, minimal tissue damage, shorter or no hospital stay. Laparoscopic approach is safe for the treatment of gallstone disease and now it is gaining clinical acceptance in various patient populations. Small incision cholecystectomy may serve as a surgical alternative for laparoscopic cholecystectomy [2]. As laparoscopic techniques are advancing, physicians are trying their best try to surgery as minimally invasive as possible. Single incision laparoscopic surgery is one of such procedures totally transumbilical single-port surgery, laparoendoscopic single-site surgery, or single incision multiport laparoendoscopic surgery. The second type of procedure is called natural orifice trans luminal endoscopic surgery, an approach that makes use of one of the body's existing orifices for abdominal access [3].

Some protective factors should be suggested to prevent the formation of gallbladder disease. Essential avoidance would be the hindrance of development of stones in subjects who have not recently had gallstones. The procedure for forestalling cholesterol gallstone arrangement ought to be focused to the fundamental pathogenetic system. Most importantly, cholesterol super saturation involves stoutness, consequently it ought to be drawn closer by lessening BMI in corpulent individuals and by bringing down fatty oil serum focuses. Fast weight reduction in the large ought to be supplanted by a steady weight reduction supported over an extensive stretch. The drawn-out various weight cycles ought to be kept away from. Sped up nucleation and bodily fluid emission in the gallbladder may be decreased by prostaglandin inhibitors. Headache medicine, in the portion of four tablets every day, was appeared to forestall gallstone arrangement in fast weight decrease patients. However, it was not affirmed that people consistently taking ibuprofen have a lower rate of gallstones. Gallbladder balance ought to be drawn closer by a customary eating design, staying away from extensive stretches between dinners. Normal exercise, as well as working with weight control, alone or in mix with abstaining from excessive food intake, improves a few metabolic anomalies identified with both stoutness and cholesterol gallstones [4].

Obesity is one of the emerging conditions in our population. Gallstones are composed of cholesterol. It is postulated that gallstones are associated with the deposition of cholesterol. The first line imaging modality is ultrasound for the diagnosis of cholelithiasis. If gallstones are correlated with BMI, it will open up a new vista for further research.

\section{METHOD AND MATERIALS}

In Yaqoob Ultrasound Clinic, PindiBhattian, Pakistan, a cross sectional analytical study was conducted. All included patients were inquired regarding variables like Age, Height, Weight, Presences of calculus, The thickness of gall bladder wall, and location of gall stones on grey scale ultrasound findings. Patients were asked to lie down and expose their abdomen; grey scale abdominal ultrasound was performed of these patients. All variables mentioned above for each patient was recorded and maintain in their individual case record form (CFR). Data was collected during allocated period.
Statically package SPSS version 2.4 was used to check the collected data and to organize and compile results.

\section{RESULTS}

According to results out of total 160 patients, 105 were females and 55 were male. Out of 160 patients $150(93.8 \%)$ were present with gall stones and $10(6.3 \%)$ were having no gall stone. In total 61 patients $(38.1 \%)$ were having single all stone whereas 89 (55.6\%) were diagnosed with multiple gall stones. According to the results, minimum age was 18 years and maximum was 80 years.

This table shows the number of male and female patients involved in the study. 105 females were examined and 55 were male. Total number of patients were 160 .

TABLE I: GENDER

\begin{tabular}{ccc}
\multicolumn{3}{c}{ TABLE I: GENDER } \\
\hline \hline Variables & Percent & Frequency \\
\hline Male & 34.4 & 55 \\
Female & 65.5 & 105 \\
Total & 100 & 160 \\
\hline \hline & & \\
\hline \hline Variables & TABLE II: GENDER & Frequency \\
\hline \hline Normal & Percent & 53 \\
Obese & 33.4 & 37 \\
Overweight & 23.1 & 70 \\
\hline \hline
\end{tabular}

\section{DISCUSSION}

Our study was designed to compare the body mass index (BMI) with cholelithiasis. On the basis of diagnostic performance and comparison of sonographic findings we can say that BMI and cholelithiasis are in direct relation with each other and ultrasonography is quite a reliable method to diagnose cholelithiasis

In current study, attempt was made to compare gall stones with body mass index. Data was collected according to variables like age, gender, height, weight, and BMI. Patient were asked to lie down and expose their abdomen. Abdominal ultrasound was performed on these patients, grey scale. The patients came for abdominal ultrasound in groups. Any unusual findings of the gall bladder were noted.

Data of 160 patients was collected out of which 105 were males and 55 were females. Out of 160 patients, $150(93.8 \%)$ patients were with cholelithiasis, whereas in $10(6.3 \%)$ patients there were no gall stones. Out of total number of 150 patients $89(55.6 \%)$ patients had multiple gall stones whereas $61(38.1 \%)$ patients had one single gall stone. The maximum age of patients was 80 years, and the minimum was 18 years.

Gallstones has become increasingly common and observed in all age groups and the incidence increases with age. Gallstones mostly remain asymptomatic. Symptoms usually appear within 5 to 20 years of diagnosis [5]. Incidence of cholelithiasis is high in female as described in most of the studies but the ratio was bit low, 3:1. There is well known saying about cholelithiasis being common in female who are fertile, around forty year of age and obese. Sex hormones are responsible for more prevalence in female patients [6].

Another study with a larger sample size $(n=2066)$ conducted in Hyderabad also showed that the younger 
patients presented with cholelithiasis in Pakistan as compared to the western countries [7]. In our study data of 13 young patients was taken and most of them were suffering from cholelithasis.

A study conducted by Imran Khan total of 134 patients were included. Female were more $(n=110$ - 82.1\%) as compared to male $(\mathrm{n}=24-17.9 \%)$. Age varied from 17 year to 65 year with a mean of 42 year. Majority $(n=94-70 \%)$ of patients were above 35 year of age. Most of the patients were in healthy group $(n=70-52 \%)$ according to the BMI classification. Overweight and obese patients were only 52 (38\%). The complete blood count (CBC) and liver function tests were found to be within normal limits. One patient of 16 year of age had hemolytic disease and was excluded from the study. Most of the patients presented with multiple stones and single stone was present in only $12(9 \%)$ patients [8]. If we compare our studies with these results 55.6 percent of the patients are presented with multiple gall stones and only 38.1 percent are presented with single stone in their gall bladder.

A study conducted in India with the mean of BMI 23.3+4.84 [9]. Females having the lower mean value of BMI as compared to the male and probable explanation is that the female sex hormones also involved in development of gallstones. Similar findings were observed in another study with a sample size of over 11000 [10]. BMI grouping in accordance with WHO criteria showed that most of the patients with cholelithiasis were from the underweight and normal weight group (61\%). All the underweight patients $(n=12)$ were females and among them six were below the age of 20 years. Previous studies have reported inconsistent associations of BMI or relative weight with gallstone disease in male populations. In a case-control study in Australia, [11] there was virtually no difference in BMI between gallstones cases and either hospital or community controls in men, while there was a positive association between BMI and gallstones in women. Another case-control study in The Netherlands showed a positive association in both men and women [12]. Two prospective studies found a positive association for relative weight or BMI with clinical gallstone disease in men and women separately in Framingham, USA [13]. Most of the studies done previously showed association of rising BMI with the development of gallstones and limited work was found to look into the reason of gallstone in patients with normal BMI. A community-based study conducted in India failed to show an association of BMI with cholelithiasiss [14]. The study conducted by our group shows that out of 160 total patients 105 were females and 55 were males and the percentage of gall stones in females is 75.6 percent and in males this percentage is 24.4. This supports the research work which proves that females are more likely to have gall stones.

The presented risk factors of cholelithiasis in women and men allow determination of groups being at high-risk $f$ this disease. Health promoting behaviors in these subjects should tend towards body mass normalization, promotion of healthy diet, and active leisure.

\section{CONCLUSION}

Our study concluded that obesity is one of the major risk factors for gall stones. Obesity is usually more common in females, so they are more likely to have gall stones and overweight patients were more prone to GB stones.

\section{REFERENCES}

[1] A. Littlefield and C. Lenahan "Cholelithiasis: presentation and management” Journal of Midwifery \& Women's Health, 64(3):289-97, 2019.

[2] C. F. BElloW, D. H. BErGEr and R. A. Crass, "Management of gallstones" American Family Physician, 72(4):637-42, 2005.

[3] S. Abraham, H. G. Rivero, I. V. Erlikh, L. F. Griffith and V. K. Kondamudi, "Surgical and nonsurgical management of gallstones" American Family Physician, 89(10):795-802, 2014.

[4] M. Acalovschi, "Cholesterol gallstones: from epidemiology to prevention” Postgraduate Medical Journal, 1;77(906):221-9, 2001.

[5] G. E. Njeze, "Gallstones" Niger J Surg, 19:49-55, 2013.

[6] Z. Sachdeva, Z. Khan, M. A. Ansari, N. Khalique and A. Anees, "Lifestyle and gallstone disease: scope for primary prevention." Indian Journal of Community Medicine, 36:263-7, 2011.

[7] N. A. Channa, F. D. Khand, M. I. Bhanger and M. H. Leghari, "Surgical incidence of cholelithiasis in Hyderabad and adjoining areas" (Pakistan). Pakistan Journal of Medical Sciences, 20:13-7, 2014.

[8] I. Khan, T. Ahmed, M. M. Iqbal, M. I. Khan, S. H. Shah and S. Perveen, "Relationship of BMI and age with cholelithiasis" Journal of Surgery Pakistan, 22(3):101-4, 2017.

[9] A. Das, A. Saikia, A. Saikia and R. Baruah," Body mass index - a predictor of gall stone disease" Indian Journal of Basic and Applied Medical Research, 5:521-7, 2015.

[10] B. Kharga, B. K. Sharma, V. K. Singh, K. Nishant, P. Bhutia, R. Tamang and N. Jain, "Obesity not necessary, risk of symptomatic cholelithiasis increases as a function of BMI,"Journal of Clinical and Diagnostic Research, 10:28-32, 2016.

[11] R. K. R. Scragg, A. J. McMichael and P. A. Baghurst, "Diet, alcohol and relative weight in gall stone disease: a case-control study" British Medical Journal (Clinical research ed.), 288(6424): 1113-1119, 1984.

[12] C. Thijs, P. Knipschild and P. Leffers "Is gallstone disease caused by obesity or by dieting" American Journal of Epidemiology, 135(3): 274280, 1992.

[13] G. D. Friedman, W. B. Kannel and T. R. Dawber, "The epidemiology of gallbladder disease: observations in the Framingham study" Journal of Chronic Diseases, 19(3): 273- 229, 1996.

[14] V. Singh, B. Trikha, C. Nain, K. Singh, S. Bose, "Epidemiology of gallstone disease in Chandigarh: A community-based study" Journal of Gastroenterology and Hepatology, 16(5): 560-563, 2001. 